\section{BMJ Open \\ Respiratory \\ Research}

\title{
Antineutrophil cytoplasmic antibody-positive conversion and microscopic polyangiitis development in patients with idiopathic pulmonary fibrosis
}

\author{
Naho Kagiyama, ${ }^{1}$ Noboru Takayanagi, ${ }^{1}$ Tetsu Kanauchi, ${ }^{2}$ Takashi Ishiguro, ${ }^{1}$ \\ Tsutomu Yanagisawa, ${ }^{1}$ Yutaka Sugita ${ }^{1}$
}

\section{ABSTRACT}

Background: Increasing evidence indicates that antineutrophil cytoplasmic antibody (ANCA)-positive conversion occurs in patients initially diagnosed with idiopathic pulmonary fibrosis (IPF) and as a result, some of these patients develop microscopic polyangiitis (MPA). However, the incidence density of these patients is not well known.

Objectives: To explore the incidence of ANCA-positive conversion and development of MPA during the disease course in patients with IPF and to evaluate whether corticosteroid therapy reduces MPA development in patients with IPF with myeloperoxidase (MPO)-ANCA positivity at diagnosis or who later acquire MPO-ANCA positivity.

Methods: We retrospectively analysed the medical records of 504 Asian patients with IPF treated at our institution in Saitama, Japan.

Results: Of the 504 patients with IPF, $20(4.0 \%)$ had MPO-ANCA and 16 (3.2\%) had PR-3-ANCA when first evaluated. In 264 of 504 patients with IPF, ANCA was measured repeatedly and seroconversion to MPO-ANCA and PR3-ANCA occurred in 15 (5.7\%) and 14 (5.3\%) patients, respectively, and 9 of 35 patients who were either MPO-ANCA positive at IPF diagnosis or who subsequently seroconverted developed MPA. None of the nine patients who developed MPA had been previously treated with steroids. The incidence of MPA tended to be lower in patients treated than not treated with corticosteroids although this was not statistically significant.

Conclusions: Some patients with IPF with MPO-ANCA positivity at IPF diagnosis or with MPO-ANCA-positive conversion during follow-up developed MPA. Clinical trials to determine whether corticosteroid therapy can reduce MPA development and prolong survival in MPOANCA-positive patients with IPF should be considered.

\section{INTRODUCTION}

Vasculitides associated with serum positivity for antineutrophil cytoplasmic antibodies (ANCAs) are commonly recognised as

\section{KEY MESSAGES}

Antineutrophil cytoplasmic antibody (ANCA)positive conversion occurs in patients initially diagnosed with idiopathic pulmonary fibrosis (IPF) and as a result, some of these patients develop microscopic polyangiitis (MPA). However, the incidence density of these patients is not well known.

Some patients with MPO-ANCA positivity at IPF diagnosis or with MPO-ANCA-positive conversion during follow-up developed MPA.

- The incidence of MPA tended to be lower in patients treated than not treated with corticosteroids although this was not statistically significant.

ANCA-associated vasculitis. ${ }^{1}{ }^{2}$ ANCAs directed against proteinase 3 (PR3) are detected mainly in patients with granulomatosis with polyangiitis, whereas ANCAs directed against myeloperoxidase (MPO) are found predominantly in patients with microscopic polyangiitis (MPA) and eosinophilic granulomatosis with polyangiitis. $^{3} \quad$ Prevalences of MPO-ANCA and PR3-ANCA positivity in patients with MPA are reported to be $30-80 \%$ and $10-30 \%$, respectively. ${ }^{4}$ MPA with pulmonary involvement is seen in up to $30 \%$ of patients ${ }^{5}$ in whom diffuse alveolar haemorrhage with pathological capillaritis is the most common manifestation.

Nada et at ${ }^{6}$ reported three patients initially diagnosed with idiopathic pulmonary fibrosis (IPF) who developed pulmonary-renal vasculitis. An association between MPA and pulmonary fibrosis has since been demonstrated. ${ }^{7-25}$ There sometimes appears to be an association between MPO-ANCA, MPA and pulmonary fibrosis. ${ }^{15}{ }^{26}$ Pulmonary fibrosis associated with MPO-ANCA or MPA is either idiopathic or 
associated with connective tissue disease, ${ }^{27}$ and the most frequent histological or radiological pattern is that of usual interstitial pneumonia. ${ }^{15-17} 22-27$ MPO-ANCA-positive IPF is recognised as a distinct phenotype of IPF. ${ }^{28}$

At least two possibilities have been proposed for the development of pulmonary fibrosis in patients with MPO-ANCA or MPA: repeated episodes of alveolar haemorrhage due to pulmonary capillaritis could be the pathogenesis of pulmonary fibrosis, ${ }^{10}$ and MPO-ANCAs may play a direct role in the pathogenesis of pulmonary fibrosis. ${ }^{16} 29$ A third hypothesis, proposed by Tzelepis $e t a l,{ }^{17}$ states that because pulmonary fibrosis is clinically manifest at MPA diagnosis, the possibility of IPF inducing MPA cannot be entirely excluded. Thereafter, Ando et $a l^{23}$ reported that during the disease course of 61 patients with IPF, MPO-ANCA positive conversion occurred in 6 patients, of whom 2 were complicated by MPA. This implies that some ANCA-negative patients with IPF acquire ANCA positivity and then develop MPA. Taken together, these findings imply that pulmonary fibrosis is not only a consequence of MPA or of a direct role of MPO-ANCA but also that it may induce ANCA and MPA.

We thus thought that the prevalence of MPO-ANCA or PR3-ANCA positivity at IPF diagnosis and the incidence density of MPO-ANCA or PR3-ANCA-positive conversion and MPA development during follow-up should be elucidated on a large scale. Therefore, we investigated risk factors of ANCA-positive conversion and whether ANCA positivity at IPF diagnosis was associated with mortality, and evaluated whether corticosteroid therapy reduces MPA development and prolongs survival in patients with IPF with MPO-ANCA positivity at diagnosis or who later acquire MPO-ANCA positivity.

\section{METHODS \\ Participants}

From 1998 through 2012, 966 patients with IPF were treated at our institution (figure 1). Of these patients, 462 were excluded: 10 patients with MPA at IPF diagnosis, 21 with exacerbation of IPF at diagnosis, 248 with simultaneous lung cancer, 39 with simultaneous chronic pulmonary infections and 144 patients in whom ANCA measurement was unavailable. Patients with IPF and lung cancer or chronic pulmonary infections were not included as these conditions can be associated with ANCA positivity. ${ }^{30}$ Thus, 504 patients comprised the cohort of this study, and they were further divided into three cohorts according to ANCA positivity at IPF diagnosis whether ANCA had or had not been measured repeatedly (figure 1). Patients were followed up through August 2013 or until death. All patients fulfilled the criteria for IPF of the American Thoracic Society and European Respiratory Society ${ }^{31}$ or the official ATS/ERS/

966 patients with IPF were diagnosed in our hospital.

20(4.0\%) patients were MPO-ANCA positive

16(3.2\%) patients were PR3-ANCA positive.

This cohort was evaluated for the incidence density and risk factors of MPA development. Median follow-up period: 2.42 years, IQR

$1.38-4.92$ years. $\rightarrow$ three of the 20 patients with MPO-ANCA positivity developed MPA
Excluded:

248 patients with lung cancer at IPF diagnosis

39 patients with chronic pulmonary infection at IPF diagnosis

21 patients with acute exacerbation at IPF diagnosis

10 patients with MPA at IPF diagnosis

144 patients in whom ANCA was not measured

504 patients in whom ANCA was measured at IPF diagnosis.

Median follow-up periods: 3.95 years, IQR: $1.84-6.64$ years.

204 patients in whom ANCA titer was not measured repeatedly were excluded.

468 patients were ANCA negative.

This cohort was evaluated for the incidence density and risk factors of MPA development. Median follow-up period: 4.02 years, IQR: $1.92-$ 6.73 years. $\rightarrow$ Six of 468 patients with ANCA negativity developed MPA

\footnotetext{
264 patients in whom ANCA titer was measured.

This cohort was evaluated for the incidence density and risk factors of ANCA-positive conversion. Median follow-up period: 5.03 years, IQR: 3.11-8.07 years. $\rightarrow$ MPO- or PR3-ANCA-positive conversion occurred $15(5.7 \%)$ patients and $14(5.3 \%)$ patients respectively
}

Figure 1 Flow diagram of enrolment and median follow-up periods in patients with idiopathic pulmonary fibrosis (IPF), ANCA, antineutrophil cytoplasmic antibody; MPO, myeloperoxidase; PR3, proteinase 3. 
Table 1 Baseline characteristics of the study patients with IPF according to ANCA positivity at diagnosis

\begin{tabular}{|c|c|c|c|c|c|}
\hline \multirow[b]{3}{*}{ Characteristic } & \multirow[b]{3}{*}{ Total } & \multicolumn{3}{|l|}{ ANCA } & \multirow[b]{3}{*}{ p Value* } \\
\hline & & \multicolumn{2}{|l|}{ Positive } & \multirow[b]{2}{*}{ Negative } & \\
\hline & & MPO-ANCA & PR3-ANCA & & \\
\hline Number of patients & 504 & 20 & 16 & 468 & \\
\hline Male & $376(74.6 \%)$ & $11(55.0 \%)$ & $11(68.8 \%)$ & $354(75.6 \%)$ & 0.072 \\
\hline Age, years & $69.5 \pm 8.1$ & $71.4 \pm 7.6$ & $73.1 \pm 6.1$ & $69.3 \pm 8.2$ & 0.041 \\
\hline Smoker & $383(76.0 \%)$ & $11(55 \%)$ & $13(81.3 \%)$ & 359 (76.7\%) & 0.414 \\
\hline Emphysema & $131(26.0 \%)$ & $5(25.0 \%)$ & $4(25.0 \%)$ & $122(26.1 \%)$ & 1 \\
\hline$\%$ FVC predicted & $74.7 \pm 19.7$ & $68.9 \pm 23.9$ & $61.6 \pm 20.1$ & $75.3 \pm 19.3$ & 0.011 \\
\hline FEV1/FVC, \% & $81.1 \pm 10.7$ & $81.1 \pm 14.8$ & $86.7 \pm 8.9$ & $80.9 \pm 10.6$ & 0.716 \\
\hline DLCO, \% predicted & $77.0 \pm 23.4$ & $74.0 \pm 11.5$ & $62.5 \pm 20.4$ & $77.5 \pm 23.6$ & 0.124 \\
\hline WCC,$/ \mu \mathrm{L}$ & $7292 \pm 2213$ & $8440 \pm 3614$ & $7731 \pm 1729$ & $7227 \pm 2139$ & 0.078 \\
\hline $\mathrm{ESR}, \mathrm{mm} / \mathrm{h}$ & $37.8 \pm 27.9$ & $74.1 \pm 41.5$ & $51.9 \pm 22.5$ & $35.8 \pm 26.1$ & $<0.001$ \\
\hline Creatinine, mg/dL & $0.80 \pm 0.48$ & $0.73 \pm 0.15$ & $0.76 \pm 0.18$ & $0.81 \pm 0.50$ & 0.078 \\
\hline $\mathrm{CRP}, \mathrm{mg} / \mathrm{d} \dagger$ & $0.20(0.10-0.63)$ & $1.17(0.50-5.30)$ & $0.45(0.16-1.63)$ & $0.20(0.10-0.60)$ & $<0.001$ \\
\hline KL-6, IU/L† & 778 (512-1263) & 646 (389-878) & 1296 (495-2118) & 784 (519-1247) & 0.589 \\
\hline RF positive & $84(16.7 \%)$ & $14(70.0 \%)$ & $2(12.5 \%)$ & $68(14.5 \%)$ & $<0.001$ \\
\hline ANA positive & $284(56.3 \%)$ & $17(85.0 \%)$ & $12(75.0 \%)$ & $255(54.5 \%)$ & 0.016 \\
\hline Urinary blood positive & $35(6.9 \%)$ & $6(30.0 \%)$ & $1(6.3 \%)$ & $28(6.0 \%)$ & 0.143 \\
\hline Urinary protein positive & $30(6.0 \%)$ & $3(15.0 \%)$ & $0(0 \%)$ & $27(5.8 \%)$ & 1 \\
\hline
\end{tabular}

JRS/ALAT statement on IPF. ${ }^{32}$ MPA was diagnosed using the Chapel Hill consensus criteria. ${ }^{1}$ This study was approved by the institutional review board of Saitama Cardiovascular and Respiratory Center.

\section{Study design}

This was a retrospective cohort study. Clinical, radiographic, laboratory data and outcome were collected from medical records. Baseline clinical parameters were obtained within 1 month of the initial diagnosis. If these data were not obtained within this period, we considered them to be unknown. Survival status was obtained from medical records and/or telephone interviews.

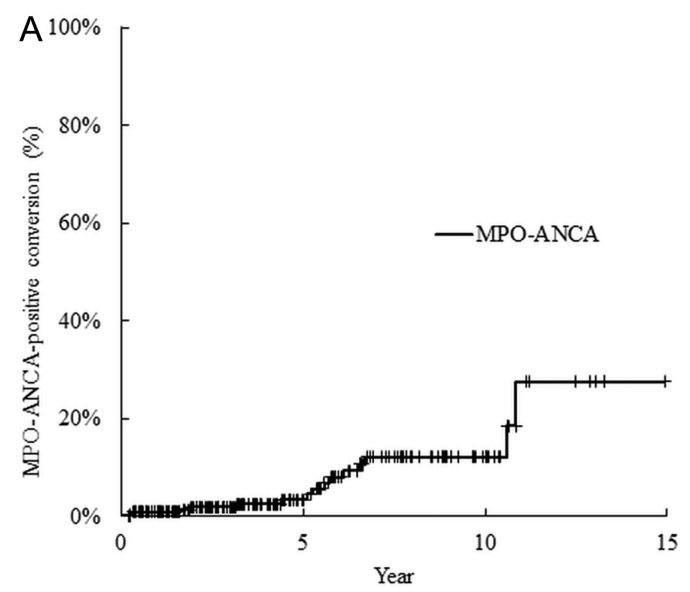

Measurement of ANCA

MPO-ANCAs and PR3-ANCAs were tested using an enzyme immunoassay: January 1998-August 1999 (NIPRO Japan), September 1999-March 2002 (BIO-RAD, USA), April 2002-March 2012 (Medical and Biological Laboratories, Japan) and April 2012 onward (Phadia Laboratory Systems, Japan).

\section{Statistical analysis}

Categorical baseline characteristics are summarised by frequency and per cent, and continuous characteristics are reported as the mean $\pm \mathrm{SD}$ or median and IQR as appropriate. Group comparisons were made using a

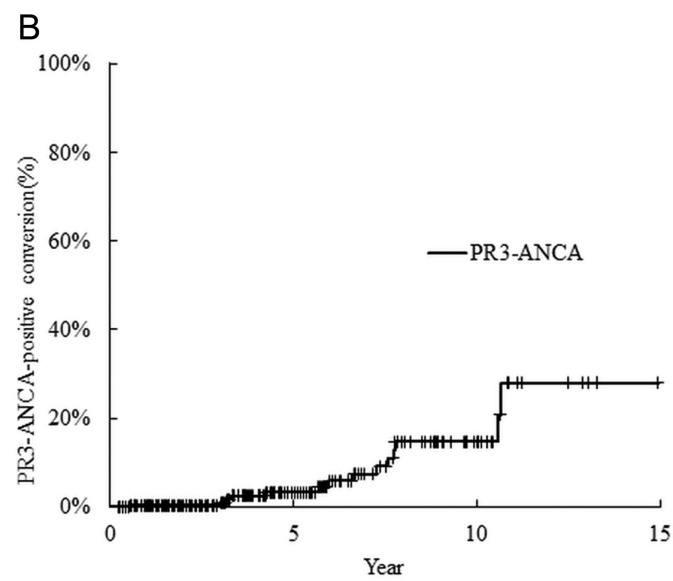

Figure 2 Kaplan-Meier curves for the time until myeloperoxidase-antineutrophil cytoplasmic antibody (MPO-ANCA)-positive conversion (A: MPO-ANCA, B: proteinase 3 [PR3]-ANCA) in patients with idiopathic pulmonary fibrosis. 
student t test, Wilcoxon rank-sum tests, or Fisher's exact test, as appropriate. ANCA-positive conversion and MPA diagnosis were estimated by Kaplan-Meier analysis. Survival was evaluated using a Kaplan-Meier curve and compared between groups using log-lank tests. Cox regression analysis was used to determine whether the following factors increased the risk of ANCA-positive conversion: sex, age, smoking history, emphysema, forced vital capacity (FVC), forced expiratory volume in $1 \mathrm{~s} \mathrm{(FEV1)/FVC} \mathrm{ratio,} \mathrm{lung} \mathrm{diffusion} \mathrm{capacity} \mathrm{for} \mathrm{carbon}$ monoxide (DLCO), white cell count, erythrocyte sedimentation rate (ESR), $\mathrm{C}$ reactive protein, serum creatine, urine protein, urine blood, rheumatoid factor, antinuclear antibody and Krebs von den Lungen-6 (KL-6). Rheumatoid factor $\geq 20 \mathrm{IU} / \mathrm{mL}$ and antinuclear antibody $\geq 1 / 80$ were considered to indicate positivity. Emphysema was considered present if areas of low attenuation were present on high-resolution CT images. Cox regression analysis was used to determine whether the following factors increased patient mortality: sex, age, smoking history, emphysema, FVC, FEV1/FVC, DLCO, MPO- or PR3-ANCA positivity, white cell count, ESR, C reactive protein, serum creatine, urine protein, urine blood, rheumatoid factor, antinuclear antibody and KL-6. In all analyses, a $p$ value of $<0.05$ was considered to be statistically significant. We conducted all statistical analyses with SAS V.9.2 (SAS Institute, Cary, North Carolina, USA).

\section{RESULTS}

\section{Patient characteristics and ANCA positivity at IPF diagnosis}

Of the 504 patients, 20 (4\%) were MPO-ANCA positive and $16(3.2 \%)$ were PR3-ANCA positive (table 1) at diagnoses. All of these patients were Asian. The ANCA-positive patients were older than the ANCA-negative patients. ESR and $\mathrm{C}$ reactive protein values were higher and $\mathrm{FVC}$ was lower in the ANCA-positive versus ANCA-negative patients. Rheumatoid factor positivity and antinuclear antibody positivity were more likely to be seen in the ANCA-positive patients than in the ANCA-negative patients.

\section{Incidence density of ANCA-positive conversion}

During the disease course, MPO-ANCA or PR3-ANCA-positive conversion occurred in 15 (5.7\%) and $14(5.3 \%)$, respectively, of the 264 patients in whom ANCA was repeatedly measured over a median follow-up period of 5.03 years (IQR, 3.11-8.07 years). Thus, the incidence density of MPO-ANCA and PR3-ANCA-positive conversion was 13.10 and 12.23 cases per 1000 personyears, respectively (figure 2 ).

\section{Risk factors for MPO- or PR3-ANCA-positive conversion}

In a multivariate Cox regression hazard model, rheumatoid factor positivity was associated with MPO-ANCA-positive conversion (adjusted HR 3.435, 95\% CI 1.032 to 11.440,

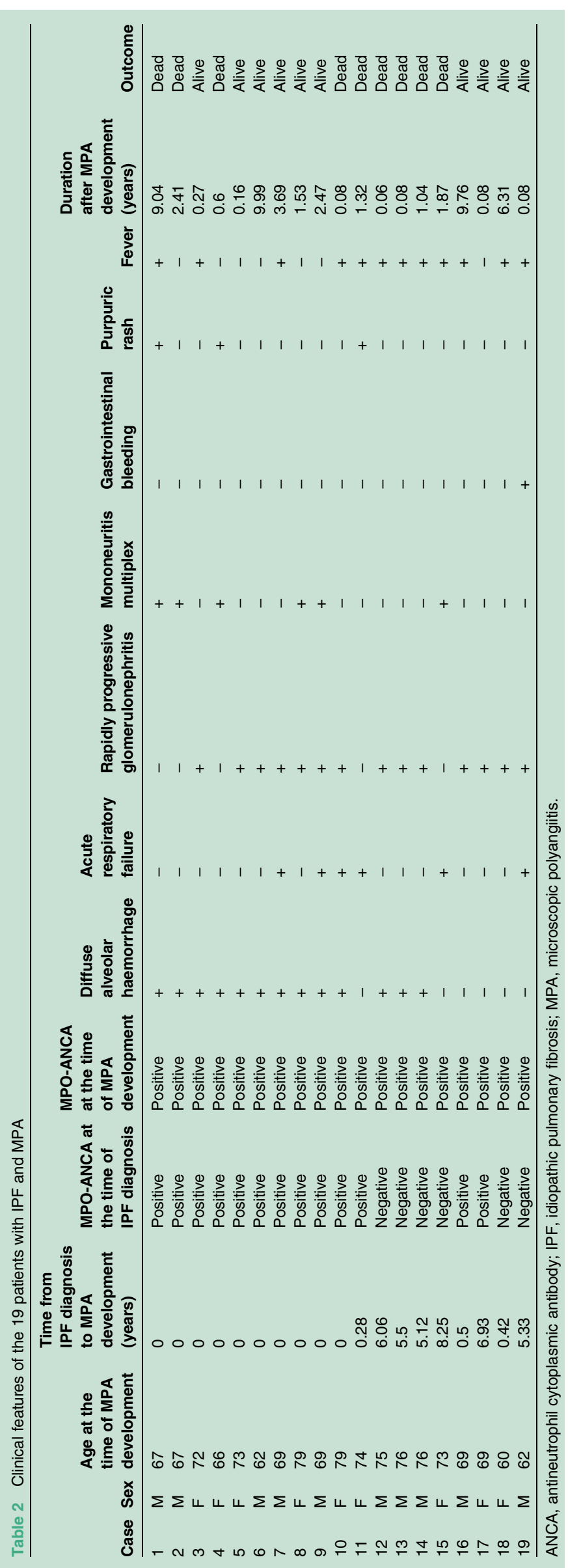




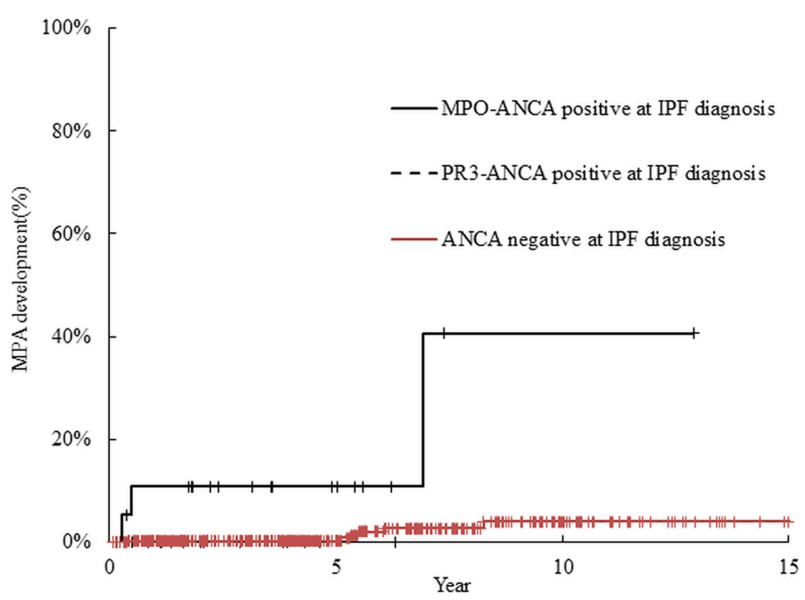

Figure 3 Kaplan-Meier curves for the time until development of microscopic polyangiitis (MPA) in patients with idiopathic pulmonary fibrosis according to antineutrophil cytoplasmic antibody (ANCA) positivity at diagnosis. The log-rank test showed the difference between ANCA-negative patients and myeloperoxidase (MPO)-ANCA-positive patients to be significant $(p<0.001)$. PR3, proteinase 3 .

$\mathrm{p}=0.044$ ), as was an ESR of $\geq 40 \mathrm{~mm} / \mathrm{h}$ (adjusted HR 3.361, $95 \%$ CI 1.100 to $10.271, \mathrm{p}=0.033)$.

\section{Incidence density of MPA development according to ANCA positivity}

Ten patients with MPA at IPF diagnosis were excluded from this analysis. Among these 10 patients $(5$ men, 5 women) with MPA at IPF diagnosis, the median age was 69 (range, 62-79) years, 7 had rapidly progressive glomerulonephritis, 10 had diffuse alveolar haemorrhage, 3 had acute respiratory failure, 4 had fever, 5 had mononeuritis multiplex and 2 had purpuric rash. In total, 9 patients in the cohort of 504 developed MPA. Among these 9 patients

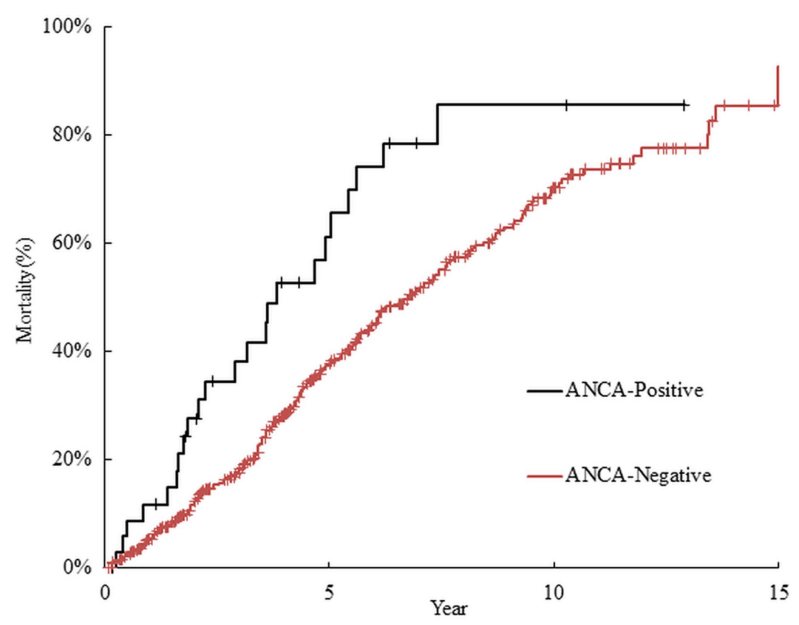

Figure 4 Kaplan-Meier survival curves of all-cause mortality according to antineutrophil cytoplasmic antibody (ANCA) positivity. The log-rank test showed the difference between ANCA-positive and ANCA-negative survival curves to be significant $(p<0.001)$.
( 5 men, 4 women), the median age was 73 (range, 60-76) years, 7 had rapidly progressive glomerulonephritis, 3 had diffuse alveolar haemorrhage, 3 had acute respiratory failure, 8 had fever, and 1 patient each had mononeuritis multiplex, gastrointestinal bleeding and purpuric rash (table 2). Three of the 20 patients with MPO positivity and none of the 16 patients with PR3-ANCA positivity at IPF diagnosis developed MPA over a median follow-up period of 2.42 years (IQR 1.38-4.92 years) (incidence density of 39.4 cases per 1000 MPO-ANCA-positive IPF person-years and 0.00 cases per 1000 PR3-ANCA-positive IPF personyears, respectively; figure 3). Of the 468 patients with ANCA negativity at IPF diagnosis, 6 developed MPA over a median follow-up period of 4.02 years (IQR 1.92-6.73 years) (incidence density of 2.75 cases per 1000 ANCA-negative IPF person-years), MPO-ANCA converted to positive in 5 patients at MPA diagnosis and 1 patient developed MPA 19 months after ANCA converted to positive. MPO-ANCA-positive patients developed MPA more frequently than did ANCA-negative patients $(\mathrm{p}<0.001)$.

\section{Mortality according to ANCA positivity at IPF diagnosis}

Of the 504 patients with IPF, death from any cause occurred in $245(48.6 \%)$ patients over a median follow-up period of 3.95 years (IQR, 1.84-6.64 years). Patients died from progression of the IPF $(35.9 \%)$, acute exacerbation of IPF $(21.0 \%)$, pneumonia $(10.1 \%)$, lung cancer $(9.3 \%)$, other pulmonary diseases $(2.4 \%)$, MPA $(1.2 \%)$, non-pulmonary diseases $(10.9 \%)$ and unknown causes $(9.2 \%)$. Five-year and 10-year mortality rates were, respectively, $61.3 \%$ and $85.7 \%$ for ANCA-positive patients, and $37.6 \%$ and $70.5 \%$ for ANCA-negative patients at IPF diagnosis. The log-rank test showed the difference between survival curves of ANCA-positive and ANCA-negative patients to be significant $(p=0.001)$ (figure 4). Five-year mortality rates of MPO-ANCA and PR3-ANCA positive patients were $51.3 \%$ and $81.7 \%$, respectively. In a multivariate Cox proportional hazard model, older age, PR3-ANCA positivity, \%FVC predicted $<70 \%, \mathrm{FEV} 1 / \mathrm{FVC} \geq 70 \%$ and DLCO $<70 \%$ were found to be negative prognostic factors (table 3). Since FEV1/ FVC $\geq 70 \%$ was an independent risk factor of IPF mortality, we compared baseline parameters of patients with IPF whose $\mathrm{FEV} 1 / \mathrm{FVC}$ ratio was $\geq 70 \%$ and $<70 \%$. In comparison with patients with an FEV1/FVC $\geq 70 \%$, patients with an FEV1/FVC $<70 \%$ were significantly more frequently male $(90.2 \%$ vs $72.5 \%, \mathrm{p}=0.006)$, smokers $(94.1 \%$ vs $75.3 \%, \mathrm{p}<0.001)$, had emphysema $(70.6 \%$ vs $21.7 \%, \mathrm{p}<0.001)$ and had higher $\% \mathrm{FVC}$ predicted $(89.00 \pm 18.43$ vs $72.74 \pm 18.97 \mathrm{~mL}, \mathrm{p}<0.001)$.

\section{MPA development and mortality in patients with positive and positively-converted MPO-ANCA according to corticosteroid therapy before MPA onset}

In this cohort of patients with IPF, a total of 35 patients were positive for MPO-ANCA (20 at IPF diagnosis and 15 subsequent seroconversion). Among this retrospective cohort of patients with IPF from 1998 to 2013, some 
Table 3 Univariate and multivariate analysis of the risk of all-cause mortality

\begin{tabular}{|c|c|c|c|c|c|c|}
\hline \multirow[b]{2}{*}{ Variable } & \multicolumn{3}{|c|}{ Univariate cox regression } & \multicolumn{3}{|c|}{ Multivariate cox regression final model } \\
\hline & Crude HR & $95 \% \mathrm{Cl}$ & p Value & Adjusted HR & $95 \% \mathrm{Cl}$ & p Value \\
\hline \multicolumn{7}{|l|}{ ANCA } \\
\hline Negative & Reference & - & - & Reference & - & - \\
\hline MPO-ANCA positive & 1.647 & 0.959 to 2.829 & 0.071 & 1.480 & 0.836 to 2.619 & 0.178 \\
\hline PR3-ANCA positive & 2.987 & 1.523 to 5.859 & 0.001 & 2.415 & 1.225 to 4.761 & 0.011 \\
\hline \multicolumn{7}{|l|}{ Sex } \\
\hline Female & Reference & - & - & & & \\
\hline Male & 0.836 & 0.625 to 1.118 & 0.226 & & & \\
\hline \multicolumn{7}{|l|}{ Age } \\
\hline$<65$ years & Reference & - & - & Reference & - & - \\
\hline$\geq 65$ years & 1.757 & 1.307 to 2.363 & $<0.001$ & 1.694 & 1.253 to 2.292 & $<0.001$ \\
\hline \multicolumn{7}{|l|}{ Smoking status } \\
\hline Never smoker & Reference & - & - & & & \\
\hline Ex/current smoker & 0.660 & 0.492 to 0.887 & 0.006 & & & \\
\hline \multicolumn{7}{|l|}{ Emphysema } \\
\hline None & Reference & - & - & & & \\
\hline Some & 0.855 & 0.647 to 1.130 & 0.272 & & & \\
\hline \multicolumn{7}{|l|}{$\% F V C$ predicted } \\
\hline$\geq 70 \%$ & Reference & - & - & Reference & - & - \\
\hline$<70 \%$ & 2.961 & 2.214 to 3.958 & $<0.001$ & 2.457 & 1.810 to 3.334 & $<0.001$ \\
\hline Unknown & 1.830 & 1.312 to 2.552 & $<0.001$ & 0.060 & 0.007 to 0.532 & 0.012 \\
\hline \multicolumn{7}{|l|}{ FEV1/FVC, \% } \\
\hline$\geq 70 \%$ & Reference & - & - & Reference & - & - \\
\hline$<70 \%$ & 0.526 & 0.331 to 0.838 & 0.007 & 0.560 & 0.347 to 0.920 & 0.017 \\
\hline Unknown & 1.173 & 0.865 to 1.592 & 0.305 & 18.379 & 2.137 to 158.081 & 0.008 \\
\hline \multicolumn{7}{|l|}{ DLCO, \% predicted } \\
\hline$\geq 70 \%$ & Reference & - & - & Reference & - & - \\
\hline$<70 \%$ & 1.920 & 1.344 to 2.743 & $<0.001$ & 1.586 & 1.099 to 2.291 & 0.014 \\
\hline Unknown & 2.125 & 1.570 to 2.876 & $<0.001$ & 1.954 & 1.377 to 2.773 & $<0.001$ \\
\hline \multicolumn{7}{|l|}{ WCC } \\
\hline$<10000 / \mu \mathrm{L}$ & Reference & - & - & & & \\
\hline$\geq 10000 / \mu \mathrm{L}$ & 1.657 & 1.157 to 2.373 & 0.006 & & & \\
\hline Unknown & 0.352 & 0.111 to 1.121 & 0.077 & & & \\
\hline \multicolumn{7}{|l|}{ ESR } \\
\hline$<40 \mathrm{~mm} / \mathrm{h}$ & Reference & - & - & & & \\
\hline$\geq 40 \mathrm{~mm} / \mathrm{h}$ & 1.627 & 1.202 to 2.203 & 0.002 & & & \\
\hline Unknown & 1.289 & 0.949 to 1.751 & 0.104 & & & \\
\hline \multicolumn{7}{|l|}{ Creatinine } \\
\hline$<1.0 \mathrm{mg} / \mathrm{dL}$ & Reference & - & - & & & \\
\hline$\geq 1.0 \mathrm{mg} / \mathrm{dL}$ & 1.178 & 0.838 to 1.656 & 0.345 & & & \\
\hline Unknown & 0.701 & 0.326 to 1.511 & 0.365 & & & \\
\hline \multicolumn{7}{|l|}{ CRP } \\
\hline$<1.0 \mathrm{mg} / \mathrm{dL}$ & Reference & - & - & & & \\
\hline$\geq 1.0 \mathrm{mg} / \mathrm{dL}$ & 1.243 & 0.916 to 1.688 & 0.163 & & & \\
\hline Unknown & 0.542 & 0.276 to 1.065 & 0.076 & & & \\
\hline \multicolumn{7}{|l|}{ KL-6 } \\
\hline$<1000 \mathrm{IU} / \mathrm{L}$ & Reference & - & - & & & \\
\hline$\geq 1000 \mathrm{IU} / \mathrm{L}$ & 1.929 & 1.426 to 2.610 & $<0.001$ & & & \\
\hline Unknown & 0.836 & 0.604 to 1.158 & 0.282 & & & \\
\hline \multicolumn{7}{|l|}{ ANA } \\
\hline Negative & Reference & - & - & & & \\
\hline Positive & 1.313 & 0.979 to 1.761 & 0.069 & & & \\
\hline Unknown & 0.705 & 0.447 to 1.112 & 0.132 & & & \\
\hline Rheumatoid factor & & & & & & \\
\hline Negative & Reference & - & - & & & \\
\hline Positive & 1.156 & 0.831 to 1.610 & 0.390 & & & \\
\hline Unknown & 0.649 & 0.454 to 0.928 & 0.018 & & & \\
\hline
\end{tabular}


Table 3 Continued

\begin{tabular}{|c|c|c|c|c|c|c|}
\hline \multirow[b]{2}{*}{ Variable } & \multicolumn{3}{|c|}{ Univariate cox regression } & \multicolumn{3}{|c|}{ Multivariate cox regression final model } \\
\hline & Crude HR & $95 \% \mathrm{Cl}$ & p Value & Adjusted HR & $95 \% \mathrm{Cl}$ & p Value \\
\hline \multicolumn{7}{|l|}{ Urinary blood } \\
\hline Negative & Reference & - & - & & & \\
\hline Positive & 1.067 & 0.674 to 1.687 & 0.782 & & & \\
\hline Unknown & 0.737 & 0.565 to 0.962 & 0.025 & & & \\
\hline \multicolumn{7}{|c|}{ Urinary protein } \\
\hline Negative & Reference & - & - & & & \\
\hline Positive & 1.066 & 0.610 to 1.865 & 0.822 & & & \\
\hline Unknown & 0.734 & 0.566 to 0.951 & 0.020 & & & \\
\hline
\end{tabular}

patients received corticosteroid treatment, although this is no longer recommended in the international guidance published in 2012. ${ }^{32}$ Among the 35 MPOANCA-positive patients, 8 had received systemic corticosteroids and none developed MPA. Among the remaining 27 MPO-ANCA-positive patients who never received steroids, 9 developed MPA. Accordingly, the incidence of developing MPA tended to be lower in patients treated with corticosteroids than in patients not treated with corticosteroids $(p=0.063)$. There was no significant difference in survival between patients treated or not treated with corticosteroids $(\mathrm{p}=0.323)$ (figure 5).

\section{DISCUSSION}

This long-term longitudinal study of a large cohort of patients with IPF resulted in five important findings. First, the incidence density of ANCA-positive conversion in patients with IPF was 13.10 cases per 1000 personyears. Second, some patients with IPF with MPO-ANCA positivity at IPF diagnosis or with MPO-ANCA positive conversion during the disease course developed MPA.
Third, rheumatoid factor positivity was a risk factor for MPO-ANCA-positive conversion, and an ESR $\geq 40 \mathrm{~mm} / \mathrm{h}$ was a risk factor for PR3-ANCA-positive conversion. Fourth, PR3-ANCA positivity at IPF diagnosis was an independent risk factor for mortality in patients with IPF. Fifth, corticosteroid therapy might reduce MPA development in patients with IPF with MPO-ANCA positivity or positive conversion.

The prevalence of MPO- and PR3-ANCA positivity in patients with IPF at diagnosis has been reported to vary between $4.9-32.1 \%$ and $2.2-3.7 \%$, respectively, ${ }^{23} 2433$ and these prevalences were comparable to ours. In patients with IPF with MPO-ANCA positivity at diagnosis, Nozu et $a l^{24}$ reported that 4 of 17 patients developed MPA, and Kang et $a l^{33}$ reported that 2 of 27 patients developed MPA. Ando $e t a t^{23}$ reported that two of six patients with MPO-ANCA-positive conversion developed MPA. These studies and ours suggest that not only patients with IPF who are MPO-ANCA positive at diagnosis but also those who convert to positive develop MPA.

Gaudin $e t a l^{19}$ reported that MPO-ANCA-associated and PR3-ANCA-associated vasculitis were both associated
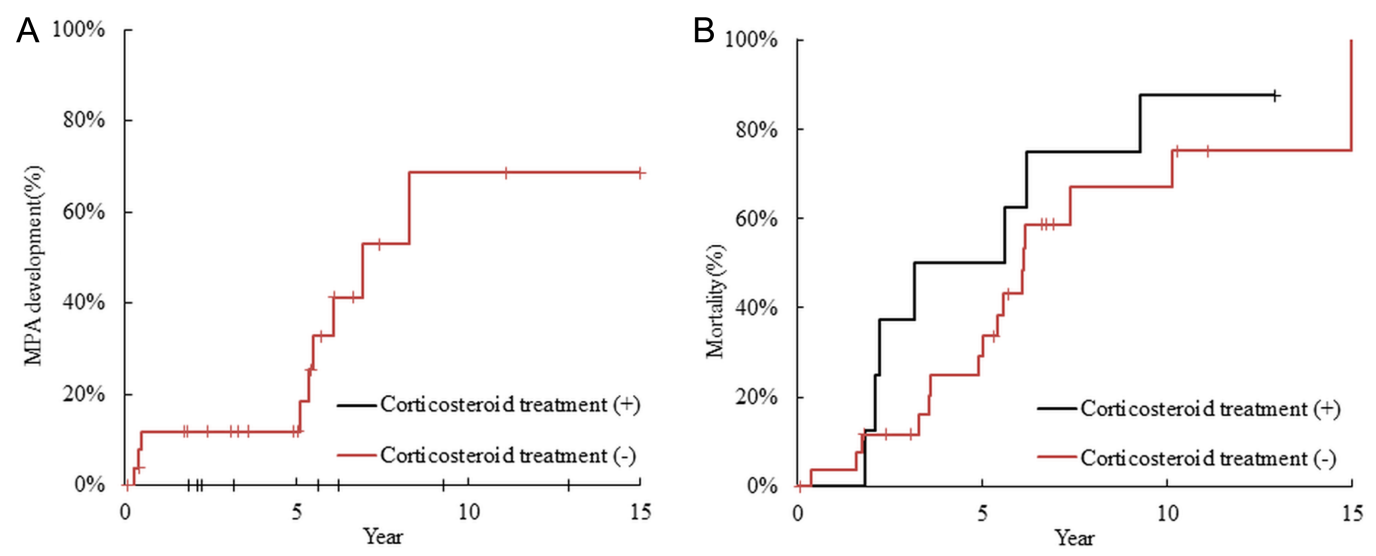

Figure 5 Kaplan-Meier curves for the time until development of microscopic polyangiitis (MPA) (A) and for survival (B) in myeroperoxidase-antineutrophil cytoplasmic antibody-positive or antibody-positively converted patients with idiopathic pulmonary fibrosis according to corticosteroid treatment. The incidence of the development of MPA tended to be lower in patients treated than not treated with corticosteroids (log-rank test: $p=0.063$ ). The difference in survival curves between patients treated and not treated with corticosteroids was not significant (log-rank test: $p=0.323$ ). 
with pulmonary fibrosis. However, subsequent reports of patients with ANCA-associated vasculitis and IPF were restricted to MPO-ANCA-positive patients. ${ }^{15-17} 22{ }^{24} \mathrm{In}$ the present study, some patients with IPF had MPO-ANCA or PR3-ANCA positivity or positive conversion. However, all of the patients who developed MPA were MPO-ANCA-positive patients. Previous studies and ours indicate that MPO-ANCA should be measured regularly in patients with IPF. Although the clinical benefit of testing for PR3-ANCA might be limited, it may be helpful because our study indicated that PR3-ANCA positivity was an independent risk factor for mortality.

Arulkumaran et $a l^{15}$ reported that of 14 patients with MPA and interstitial lung disease, 2 were diagnosed as having interstitial lung disease 6 months and 3 years, respectively, before MPA. Three of their patients presented with MPA before interstitial lung disease with a mean interval of 9.7 years, and 9 patients presented with features of interstitial lung disease and MPA concurrently. If pulmonary fibrosis develops after the onset of MPA, repeated episodes of alveolar haemorrhage due to pulmonary capillaritis could be the pathogenesis of interstitial fibrosis. Tanaka $e t a l^{25}$ reported that in nine patients with interstitial pneumonia associated with MPO-ANCA but without MPA, surgical lung biopsy showed neither capillaritis nor vasculitis. Therefore, if patients with IPF are proved to have MPO-ANCA at IPF diagnosis, two possible pathogeneses of pulmonary fibrosis may be present: MPO-ANCA is playing a direct role in the pathogenesis of pulmonary fibrosis, and pulmonary fibrosis is inducing MPO-ANCA. During the disease course, if MPO-ANCA-positive conversion occurs, IPF might be inducing MPO-ANCA and MPA.

Therapy with corticosteroids in combination with other immunosuppressants is the current mainstay of MPA treatment. ${ }^{44}$ However, the 2012 official ATS/ERS/JRS/ALAT statement recommends that patients with IPF should not be treated with corticosteroid monotherapy or combined corticosteroid and immunomodulator therapy. ${ }^{32}$ Moreover, increased risks of death and hospitalisation were observed in patients with IPF who were treated with a combination of prednisone, azathioprine and N-acetylcysteine, as compared with placebo. ${ }^{35}$ However, if patients with IPF develop MPA, patients should be treated with corticosteroids because untreated MPA is normally rapidly progressive and fatal. $^{4}$ Thus, should patients with IPF with MPO-ANCA positivity but without MPA be treated with corticosteroids? Ando et al reported that 6 of 9 MPO-ANCA-positive patients with IPF were treated with corticosteroid therapy and none developed MPA, but 2 of 3 patients not treated with corticosteroids developed MPA. ${ }^{23}$ We hypothesise that corticosteroid therapy in patients with IPF with MPO-ANCA positivity might have some benefit in reducing the development of MPA.

One limitation of this study is that it was retrospective, so some clinical and laboratory findings were not available. Second, because the decision to measure ANCA was made by referring doctors and the interval of measurement of ANCA was not unified, we could not conclude how often ANCA should be measured in patients with IPF. Finally, because the ELISA kit had been changed four times during the study period, we could not accurately assess the association of ANCA titres with MPA development.

In conclusion, the present study showed that the incidence density of ANCA-positive conversion in patients with IPF was 13.10 cases per 1000 person-years. Some patients with IPF with MPO-ANCA positivity at IPF diagnosis or with MPO-ANCA-positive conversion during the disease course developed MPA. PR3-ANCA positivity at IPF diagnosis was found to be an independent risk factor for mortality. Clinical trials to determine whether corticosteroid therapy can reduce MPA development and prolong survival in MPO-ANCA-positive patients with IPF should be considered

Acknowledgements The authors offer their sincerest thanks to Drs Youtaro Takaku and Kazuyoshi Kurashima of the Department of Respiratory Medicine, Saitama Cardiovascular and Respiratory Center, for their handling of the diagnosis and treatment of the patients with IPF.

Contributors NK performed the primary data analysis. NT had the idea for the study. NT and TK reviewed the chest radiographs and high-resolution CT scans. NK and NT wrote the manuscript. TI, TY and YS contributed to study design, interpreted the data and reviewed the manuscript. All authors approved the final version of the manuscript.

Competing interests None.

Ethics approval This study was approved by the institutional review board of Saitama Cardiovascular and Respiratory Center.

Provenance and peer review Not commissioned; externally peer reviewed.

Data sharing statement No additional data are available.

Open Access This is an Open Access article distributed in accordance with the Creative Commons Attribution Non Commercial (CC BY-NC 4.0) license, which permits others to distribute, remix, adapt, build upon this work noncommercially, and license their derivative works on different terms, provided the original work is properly cited and the use is non-commercial. See: http:// creativecommons.org/licenses/by-nc/4.0/

\section{REFERENCES}

1. Jennette JC, Falk RJ, Andrassy K, et al. Nomenclature of systemic vasculitides. Proposal of an international consensus conference. Arthritis Rheum 1994;37:187-92.

2. Watts R, Lane S, Hanslik T, et al. Development and validation of a consensus methodology for the classification of the ANCA-associated vasculitides and polyarteritis nodosa for epidemiological studies. Ann Rheum Dis 2007;66:222-7.

3. Jennette JC, Falk RJ, Bacon PA, et al. 2012 revised international chapel hill consensus conference nomenclature of Vasculitides. Arthritis Rheum 2013;65:1-11.

4. Gómez-Puerta JA, Hernández-Rodriguez J, López-Soto A, et al. Antineutrophil cytoplasmic antibody-associated vasculitides and respiratory disease. Chest 2009;136:1101-11.

5. Brown KK. Pulmonary vasculitis. Proc Am Thorac Soc 2006;3:48-57.

6. Nada AK, Torres VE, Ryu JH, et al. Pulmonary fibrosis as an unusual clinical manifestation of a pulmonary-renal vasculitis in elderly patients. Mayo Clin Proc 1990;65:847-56.

7. Becker-Merok A, Nossent JC, Ritland N. Fibrosing alveolitis predating microscopic polyangiitis. Scand J Rheumatol 1999;28:254-6.

8. Hiromura K, Nojima Y, Kitahara T, et al. Four cases of anti-myeloperoxidase antibody-related rapidly progressive glomerulonephritis during the course of idiopathic pulmonary fibrosis. Clin Nephrol 2000;53:384-9. 
9. Mansi IA, Opran A, Sondhi D, et al. Microscopic polyangiitis presenting as idiopathic pulmonary fibrosis: is anti-neutrophilic cytoplasmic antibody testing indicated? Am J Med Sci 2001:321:201-2.

10. Eschun GM, Mink SN, Sharma S. Pulmonary interstitial fibrosis as a presenting manifestation in perinuclear antineutrophilic cytoplasmic antibody microscopic polyangiitis. Chest 2003;123:297-301.

11. Birnbaum J, Danoff S, Askin FB, et al. Microscopic polyangiitis presenting as a "pulmonary-muscle" syndrome: is subclinical alveolar hemorrhage the mechanism of pulmonary fibrosis? Arthritis Rheum 2007;56:2065-71.

12. Bhanji A, Karim M. Pulmonary fibrosis-an uncommon manifestation of anti-myeloperoxidase-positive systemic vasculitis? NDT Plus 2010;3:351-3.

13. Shields O, Shah A, Mann B. Pyrexia of unknown origin and pulmonary fibrosis as a presentation of MPO-ANCA associated vasculitis. BMJ Case Rep 2011;2011. doi:10.1136/bcr.01.2011.3692

14. Eleftheriou D, Katsenos S, Zorbas S, et al. Pulmonary fibrosis presenting as an early manifestation of microscopic polyangiitis. Monaldi Arch Chest Dis 2012;77:141-4.

15. Arulkumaran N, Periselneris N, Gaskin G, et al. Interstitial lung disease and ANCA-associated vasculitis: a retrospective observational cohort study. Rheumatology (Oxford) 2011:50:2035-43.

16. Hervier B, Pagnoux C, Agard C, et al. Pulmonary fibrosis associated with ANCA-positive vasculitides. Retrospective study of 12 cases and review of the literature. Ann Rheum Dis 2009;68:404-7.

17. Tzelepis GE, Kokosi M, Tzioufas A, et al. Prevalence and outcome of pulmonary fibrosis in microscopic polyangiitis. Eur Resp $J$ 2010;36:116-21.

18. Gal AA, Salinas FF, Staton GW Jr. The clinical and pathological spectrum of antineutrophil cytoplasmic autoantibody-related pulmonary disease. A comparison between perinuclear and cytoplasmic antineutrophil cytoplasmic autoantibodies. Arch Pathol Lab Med 1994:118:1209-14.

19. Gaudin PB, Askin FB, Falk RJ, et al. The pathologic spectrum of pulmonary lesions in patients with anti-neutrophil cytoplasmic autoantibodies specific for anti-proteinase 3 and anti-myeloperoxidase. Am J Clin Pathol 1995;104:7-16.

20. Ando Y, Okada F, Matsumoto S, et al. Thoracic manifestation of myeloperoxidase-antineutrophil cytoplasmic antibody (MPO-ANCA)-related disease. CT findings in 51 patients. J Comput Assist Tomogr 2004;28:710-16.

21. Chung MP, Yi CA, Lee HY, et al. Imaging of pulmonary vasculitis. Radiology 2010;255:322-41.
22. Foulon G, Delaval P, Valeyre D, et al. ANCA-associated lung fibrosis: analysis of 17 patients. Respir Med 2008;102: 1392-8.

23. Ando M, Miyazaki E, Ishii T, et al. Incidence of myeloperoxidase anti-neutrophil cytoplasmic antibody positivity and microscopic polyangitis in the course of idiopathic pulmonary fibrosis. Respir Med 2013;107:608-15

24. Nozu T, Kondo M, Suzuki K, et al. A comparison of the clinical features of ANCA-positive and ANCA-negative idiopathic pulmonary fibrosis patients. Respiration 2009;77:407-15.

25. Tanaka T, Otani K, Egashira R, et al. Interstitial pneumonia associated with MPO-ANCA: clinicopathological features of nine patients. Respir Med 2012;106:1765-70.

26. Yamada H. ANCA: associated lung fibrosis. Semin Respir Crit Care Med 2011;32:322-7.

27. Homma S, Matsushita H, Nakata K. Pulmonary fibrosis in myeloperoxidase antineutrophil cytoplasmic antibody-associated vasculitides. Respirology 2004;9:190-6.

28. Poletti $\mathrm{V}$, Ravaglia $\mathrm{C}$, Buccioli $\mathrm{M}$, et al. Idiopathic pulmonary fibrosis: diagnosis and prognostic evaluation. Respiration 2013;86:5-12.

29. Guilpain P, Chéreau C, Goulvestre C, et al. The oxidation induced by antimyeloperoxidase antibodies triggers fibrosis in microscopic polyangiitis. Eur Respir J 2011:37:1503-13.

30. Tsiveriotis K, Tsirogianni A, Pipi E, et al. Antineutrophil cytoplasmic antibodies testing in a large cohort of unselected greek patients. Autoimmune Dis 2011;2011:626495.

31. American Thoracic Society. Idiopathic pulmonary fibrosis: diagnosis and treatment. International consensus statement. American Thoracic Society (ATS), and the European Respiratory Society (ERS). Am J Respir Crit Care Med 2000;161:646-64.

32. Raghu G, Collard HR, Egan JJ, et al. An official ATS/ERS/JRS/ ALAT statement: idiopathic pulmonary fibrosis: evidence-based guidelines for diagnosis and management. Am J Respir Crit Care Med 2011;183:788-824.

33. Kang BH, Park JK, Roh JH, et al. Clinical significance of serum autoantibodies in idiopathic interstitial pneumonia. J Korean Med Sci 2013;28:731-7.

34. Guillevin L, Durand-Gasselin B, Cevallos R, et al. Microscopic polyangiitis: clinical and laboratory findings in eighty-five patients. Arthritis Rheum 1999;42:421-30.

35. Raghu G, Anstrom KJ, King TE Jr, et al. Idiopathic Pulmonary Fibrosis Clinical Research Network, Prednisone, azathioprine, and $\mathrm{N}$-acetylcysteine for pulmonary fibrosis. $N$ Engl $J$ Med 2012;366:1968-77. 